\title{
Notices
}

\author{
IEEE Workshop on Visual Motion \\ Irvine, California \\ March 20-22, 1989
}

The analysis of visual motion is attracting increasing attention from researchers in Computer Vision, Visual Perception, and Artificial Intelligence. This workshop will bring together researchers from this broad range of disciplines to discuss current work on the representation and analysis of motion in image sequences. Sessions will be devoted to each of several aspects of motion research. The number of presentations will be limited, with the aim of increasing discussions. Papers are invited on all aspects of the analysis of human and machine vision, including: Motion detection mechanisms - Optical flow and motion correspondence • Structure from motion • Event recognition and representation $\bullet$ Temporal planning and inferences $\bullet$ Control structures for dynamic scene analysis - Uncertainty in dynamic scene analysis - Applications in navigation, object manipulation, and recognition.

Authors are encouraged to present new computational methods with experimental results, theoretical results that offer significant new insights into problem solution, or to relate experimental observations on human visual processing to the underlying computational strategies used.

Submission of papers: Three copies of the paper should be submitted to either Ellen Hildreth (Artificial Intelligence Laboratory, 545 Technology Square, Cambridge, MA 02139) or Ramesh Jain (Elect Engineering and Computer Science, The University of Michigan, Ann Arbor, MI 48109-2122) on or before July 15, 1988. The papers should not exceed 25 double-spaced pages. Authors will be notified of the decisions by October 15, 1988. Final papers will be required by the Computer Society of the IEEE on November 25, 1988.

\section{Third Symposium on Progress in Research on Brain and Behavior "Advances in Research on Cerebral Laterality Effects" Toledo, Ohio April 8-9, 1988}

The Third Symposium on Progress in Research on Brain and Behavior, sponsored by the University of Toledo Department of Psychology and the University of Toledo Alumni Foundation, will be held in the Driscoll Center for Continuing Education on the University of Toledo campus on April 8 and 9, 1988.

The theme for this symposium is Advances in Research on Cerebral Laterality Effects. Invited papers will be presented on advances in our knowledge of laterality effects with topics including research in the areas of anatomy, electrophysiology, neuropsychology, and behavior.

The participants for this symposium are M. P. Bryden, A. S. Gevins, C. R. Hamilton, C. Hardyck, J. B. Hellige, M. Kinsbourne, S. M. Kosslyn, J. Levy, W. F. McKeever, D. Molfese, J. Sergent, J. Ward, S. Witleson, and F. Wood.

For registration and other information, contact Fred Kitterle, Department of Psychology, University of Toledo, Toledo, Ohio 43606 (Phone: (419)537-2722). 